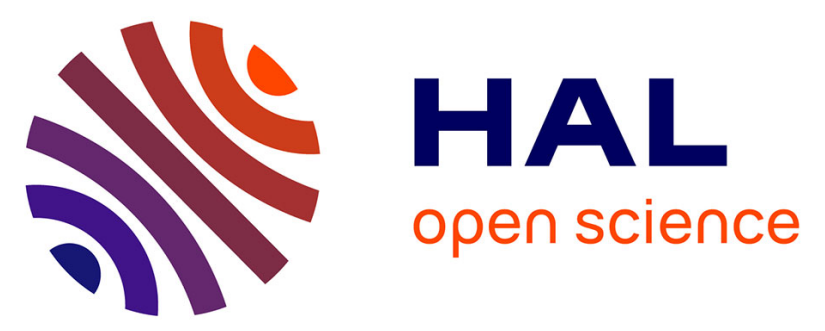

\title{
Le portage de repas à domicile : processus d'installation et d'appropriation de ce service par les personnes âgées en perte d'autonomie
}

\author{
Ségolène Fleury, Claire Sulmont-Rossé, Paul Tronchon, Stéphanie Roux, \\ Virginie van Wymelbeke-Delannoy
}

\section{To cite this version:}

Ségolène Fleury, Claire Sulmont-Rossé, Paul Tronchon, Stéphanie Roux, Virginie van WymelbekeDelannoy. Le portage de repas à domicile : processus d'installation et d'appropriation de ce service par les personnes âgées en perte d'autonomie. Nutrition Clinique et Métabolisme, 2021, 35 (2), pp.107-115. 10.1016/j.nupar.2020.10.004 . hal-03225401

\author{
HAL Id: hal-03225401 \\ https://hal.inrae.fr/hal-03225401
}

Submitted on 12 Jan 2022

HAL is a multi-disciplinary open access archive for the deposit and dissemination of scientific research documents, whether they are published or not. The documents may come from teaching and research institutions in France or abroad, or from public or private research centers.
L'archive ouverte pluridisciplinaire HAL, est destinée au dépôt et à la diffusion de documents scientifiques de niveau recherche, publiés ou non, émanant des établissements d'enseignement et de recherche français ou étrangers, des laboratoires publics ou privés.

\section{(1) (1) $\$$}

Distributed under a Creative Commons Attribution - NonCommercial - NoDerivatives 44.0 
LE PORTAGE DE REPAS A DOMICILE : PROCESSUS D'INSTALLATION ET D'APPROPRIATION DE CE SERVICE PAR LES PERSONNES AGEES EN PERTE D'AUTONOMIE

HOME-DELIVERED MEALS: SETTING UP AND APPROPRIATION PROCESSES AMONG THE OLDER ADULTS LOSING AUTONOMY

Ségolène Fleury ${ }^{1,2}$, Claire Sulmont-Rossé ${ }^{1 *}$, Paul Tronchon ${ }^{2}$, Stéphanie Roux ${ }^{2}$, Virginie Van Wymelbeke-Delannoy ${ }^{1,3}$

${ }^{1}$ Centre des Sciences du Goût et de l'Alimentation, AgroSup Dijon, CNRS, INRAE, Université de Bourgogne Franche-Comté, F-21000 Dijon, France

${ }^{2}$ Saveurs et Vie, Orly, France

${ }^{3}$ CHU Dijon Bourgogne, Unité de recherche Pôle Personnes Âgées, Dijon, France

\section{AUTEUR CORRESPONDANT}

* Claire Sulmont-Rossé

Mail : claire.sulmont-rosse@inrae.fr

\section{MOTS-CLES}

Vieillissement, portage de repas, domicile, dépendance, satisfaction

\section{KEYWORDS}

Aged, meal-on-wheel, community-dwelling, dependence, satisfaction 


\section{RESUME}

La survenue d'incapacités physiques ou cognitives peut conduire une personne âgée à déléguer certaines tâches de la vie courante à un aidant. Si la mise en place d'aides permet le maintien à domicile et est fortement plébiscité par les seniors, la perte d'autonomie constitue néanmoins un élément de rupture dans sa vie d'une personne. Objectif. Notre objectif était d'explorer les processus d'installation d'un service de livraison de repas à domicile et les mécanismes d'appropriation de ce service par les bénéficiaires. Méthode. Des entretiens semidirectifs ont été réalisés auprès de 30 bénéficiaires d'un service de livraison de repas (21 femmes ; 70-97 ans). Résultat. L'analyse thématique des entretiens a montré que la mise en place de ce service faisait souvent suite à une perte de capacités fonctionnelles, limitant la capacité des personnes à faire leurs courses. Deux dynamiques principales ont été observées : pour certains, l'installation de ce service est une étape dans l'ajustement progressif des aides aux besoins de la personne, tandis que pour d'autres l'installation du service est soudaine, souvent à la suite d'une hospitalisation. Le contexte de vie de la personne âgée et la présence de proches influencent cette dynamique. De fait, l'implication effective de la personne dans l'installation de ce service varie beaucoup d'une personne à l'autre et peut influencer la perception des repas reçus. Conclusion. La revalorisation du portage de repas à domicile passe probablement par l'introduction d'espaces de liberté dans ce service (personnalisation des prestations), au regard des trajectoires de vie très variées des bénéficiaires.

\section{ABSTRACT}

The onset of disabilities may lead older adults to delegate some daily activities to a caregiver. While a large majority of older people look forward to staying at home as long as possible, the loss of autonomy may constitute a disruptive element in a person's life. Objective. Our objective was to explore the setting-up and the appropriation processes of meals-on-wheels service among older adults. Method. Semi-directive interviews were conducted with 30 beneficiaries of meals-on-wheels service (21 women; 70-97 years old). Result. The thematic analysis of the interviews showed that the implementation of this service is often linked to a loss of functional capacity which limits older adults' ability to do their food shopping. Two main situations were observed: for some participants, the implementation of this service is one step among others within in the gradual implementation of various care services, while 
for others the implementation of a meals-on-wheels service is sudden, often following hospitalization. This dynamic is influenced by the life situation of the older person and the presence/absence of relatives. In fact, the personal involvement of an older individual in the implementation of this service varies greatly from one person to another and can influence the perception of this service. Conclusion. Meals-on-wheels service may be improved by introducing "freedom spaces" such as provision of choice or individualized delivery service, considering the life situation of each beneficiary.

\section{INTRODUCTION}

Comme dans la plupart des pays développés, la proportion de personnes âgées dans la population française est en pleine croissance (12 millions en 2005 contre des prévisions à l'horizon 2060 estimées à 24 millions). Le vieillissement de la population se caractérise notamment par une forte progression des personnes très âgés. D'ici à 2060, le nombre de personnes âgées de plus de 85 ans devrait quadrupler : 1,3 million aujourd'hui, 5,4 millions en 2060 (1). Une forte progression des personnes en perte d'autonomie (deux fois plus de personnes d'ici à 2060, soit 2,3 millions de personnes âgées dépendantes est également prédite (2,3). Face à ces chiffres, il convient de rappeler que la capacité d'accueil en institution reste limitée - environ 720000 places en 2011 et que seuls $9 \%$ des plus de 75 ans vivent dans un établissement d'hébergement pour personnes âgées (3). Pour la majorité, le maintien à domicile est largement plébiscité : 77\% des Français le revendiquent (4). Il y a donc là un véritable enjeu sociétal : développer des solutions d'aides à domicile pertinentes, c'est-à-dire adaptées aux besoins, capacités et attentes de la population âgée.

Au cours de sa vie, chaque individu est confronté à des périodes de transition qui façonnent sa trajectoire de vie. Le concept de transition envisagé ici correspond à une période de changement où l'individu est confronté à l'abandon de rôles et de statuts familiers pour en acquérir de nouveaux (5). Le vieillissement s'accompagne fréquemment de périodes de transition susceptibles de modifier l'organisation de la vie quotidienne : passage à la retraite, veuvage, survenue d'une pathologie, de handicaps. En particulier, la survenue d'incapacités physiques (difficultés à se déplacer, porter des charges...) ou cognitives (trouble de la mémoire...) peut conduire les personnes âgées à déléguer certaines tâches de la vie courante à un tiers - un aidant informel (conjoint, enfant...) ou formel (aide-ménagère, service d'aide à 
domicile, portage de repas ...). De plus, certaines personnes âgées, en particulier les femmes évoquent ne plus avoir envie de s'occuper des tâches liées à l'alimentation dont elles ont été responsable toute leur vie. Dans cette génération, les tâches ménagères et notamment celles liées à l'alimentation restent très genrées (6-8). Ces aides à domicile permettent une certaine continuité dans les habitudes de vie des personnes âgées tout en étant parfois indispensables au maintien de la personne âgée à son domicile. Pour autant, l'introduction d'une tierce personne dans l'intimité du foyer, pour soutenir ou substituer la personne âgée dans des tâches qui étaient de sa compétence, constitue un élément de rupture dans sa vie $(9,10)$. L'intimité est caractérisée par la frontière entre soi et les autres, elle peut se matérialiser par le besoin d'un «chez soi » où l'on peut laisser s'exprimer sans contrainte son identité (11). La mise en place d'une aide à domicile traduit une perte d'autonomie ; l'autonomie se référant ici à la liberté, à l'indépendance d'une personne faisant ainsi des choix par elle-même et agissant en conséquence (12).

Cardon et Gojard (2009) (13) ont proposé le terme de « dépendance culinaire » pour désigner des situations dans lesquelles des personnes âgées ne peuvent plus assurer l'approvisionnement et/ou la préparation des repas et sont conduites à les déléguer à un tiers. Il existe différents degrés de dépendance culinaire : aide pour les courses, aide pour la préparation des repas, voire aide pour la prise alimentaire. P. Cardon $(2007,2010)(14,15)$ a mis en évidence différentes formes de délégation, en fonction du statut des aidants et de la relation aidant-aidé. La délégation sous contrôle (ou subordination) correspond au cas où l'aidant « fait pour » la personne âgée mais selon la volonté de cette dernière. La complémentarité correspond au cas où l'aidant et l'aidé « font ensemble ». La gestion des tâches alimentaires est alors le fruit de compromis entre les deux parties. Enfin, la délégation totale (ou substitution) correspond au cas où l'aidant « fait à la place de » la personne âgée. Si dans les deux premières situations, la personne âgée garde une part de contrôle sur ses pratiques alimentaires, elle y renonce complètement dans la troisième situation. La délégation des activités liés à l'alimentation n'est pas sans conséquence sur l'alimentation des personnes âgées. Ainsi, Gojard \& L'huissier, (2003) ont montré que la délégation des courses à une aide-ménagère entraînait une diminution de la variété alimentaire (16). Maitre et al., (2014) ont par ailleurs montré que $46 \%$ des personnes âgées vivant à domicile et bénéficiant d'une aide pour les activités alimentaires étaient dénutries ou à risque de dénutrition contre 
seulement $16 \%$ des personnes âgées bénéficiant d'une aide pour les activités quotidiennes autres qu'alimentaires (ménage, toilette...) (17).

Dans cet article, nous nous intéresserons au cas particulier de la livraison de repas à domicile. Ce service concerne 80000 personnes en France (18) et est proposé par de nombreuses collectivités ou associations dans le cadre de l'action sociale. De fait, $66 \%$ des communes de 5000 à 20000 habitants proposent un service de portage de repas aux personnes âgées les plus démunies et/ou dépendantes (19). La mise en place d'un portage de repas à domicile dans le cadre de l'action sociale dépend de trois conditions :

- le niveau de dépendance (incapacité à faire soi-même les courses et la cuisine) ;

- les revenus (prise en charge de tout ou partie du coût du portage par les mairies, les conseils départementaux et éventuellement par les caisses de retraite en fonction du revenu de la personne);

- l'âge (être âgé d'au moins 65 ans ou d'au moins 60 ans pour une personne inapte au travail).

Dans le cas de l'action sociale, le service de portage est réalisé soit par une cuisine centrale la ville, ou délégué à une entreprise privée dans le cadre d'une prestation de service. Mais le portage de repas peut aussi être réalisé par les entreprises privées en vente directe auprès d'un marché de personnes âgées souvent plus aisées.

L'objectif de notre étude était d'explorer les processus d'installation d'un service de livraison de repas à domicile et les mécanismes d'appropriation de ce service par les bénéficiaires. En particulier, nous nous sommes intéressés à la "capacité à agir", que ce soit en amont de l'installation (prise d'information, démarches...) ou en aval. Cette "capacité à agir" fait écho à la notion de "human agency" décrite par les théoriciens du parcours de vie. Cette notion se réfère aux « efforts d'exercer une influence sur l'orientation de sa trajectoire de vie » (20). 


\section{PARTICIPANTS ET MÉTHODES}

\subsection{Les participants}

Trente et une personnes âgées de 70 ans et plus bénéficiant d'un portage de repas à domicile ont été recrutées entre février 2018 et juin 2019 à Paris et dans sa banlieue. Parmi ces volontaires, 16 étaient des bénéficiaires du service de portage de repas proposé par le Centre d'Action Sociale de la Ville de Paris (CASVP). Les 15 autres volontaires étaient des clients directs de la même société que le prestataire du CASVP. La principale différence entre ces deux types d'usagers réside dans le coût de la prestation : les tarifs de la mairie de Paris sont proportionnels aux revenus des usagers tandis que le tarif pour la clientèle directe est fixe. L'offre proposée aux clients directs est plus large et plus personnalisable que celle des clients CASVP.

Le protocole a été déposé et approuvé par un comité d'éthique (CPP EST1 2015/24 - IDRCB \# 2015-A01324-45). Conformément à la législation en vigueur et aux règles déontologiques, les volontaires ont reçu une note d'information et signé un consentement éclairé avant leur participation à l'étude. Toutes les données recueillies ont été rendues anonymes.

\subsection{Déroulement des entretiens}

Lors d'une première visite à domicile, l'étude était présentée aux participants et leur consentement était recueilli. Cette première visite permettait de recueillir les données suivantes : données socio-démographiques (âge, sexe, statut marital, niveau d'étude, autoperception de sa situation financière, nombre d'enfants), caractéristiques du portage (nombre de repas livrés par semaine, prix du repas, date de début du portage), statut cognitif (MiniMental State Examination - MMSE (21).

Lors d'une seconde visite à domicile, chaque volontaire participait à un entretien d'environ une heure à domicile. Les entretiens ont été enregistrés au moyen d'un dispositif audio et intégralement retranscrits. Dans un premier temps, l'histoire de l'installation du portage de repas ne pouvant être dissocié de la trajectoire de vie, l'entretien commençait avec la grille Ageven (22). Cette grille permettait de retracer dans l'ordre chronologique les événements marquants de la vie du participant concernant sa vie familiale (lieux de résidence, évènements familiaux...), sa vie professionnelle (activités exercées, retraite...) et sa santé (maladies 
graves, hospitalisations...). Dans un second temps, le participant était interrogé à l'aide d'une grille d'entretien spécialement développée pour cette étude, comportant quatre thèmes :

- implication dans l'acte alimentaire de la personne au début de sa vie de retraité ;

- difficultés rencontrées par la personne dans les activités quotidiennes liées à son alimentation ;

- histoire de l'installation du portage de repas ;

- perception et attentes de la personne vis-à-vis de son service de livraison de repas à domicile.

Cette deuxième partie de l'entretien s'appuyait sur la chronologie des évènements retracés dans un premier temps à l'aide de la grille Ageven. Les entretiens ont été menés de manière à laisser une certaine liberté de parole à l'enquêté. Néanmoins, tous les thèmes de la grille d'entretien étaient systématiquement abordés de manière à permettre la comparaison des discours et d'en faciliter l'analyse.

\subsection{Méthode d'analyse}

L'analyse des discours a permis d'explorer les différents processus de mise en place d'un portage de repas à domicile et de s'imprégner du vécu de cet évènement par les personnes âgées et leur perception de ce service. Au cours d'une première étape, l'histoire de l'installation du portage de repas à domicile a été résumée pour chaque participant. Au cours d'une seconde étape, une analyse thématique des discours a été réalisée afin d'identifier les concepts émergeant pour chacun des quatre thèmes abordés au cours de l'entretien. Enfin, trois expérimentateurs extérieurs à l'étude ont indépendamment évaluer la satisfaction des participants vis-à-vis de leur prestation de portage. Après la lecture des verbatims du IV ${ }^{\text {ème }}$ thème les participants étaient catégorisés « Très satisfait / Satisfait / Pas satisfait / Pas du tout satisfait»par les expérimentateurs. Les points forts et les points faibles de la prestation, rapportés par chaque participant, ont également été listés. 


\section{RÉSULTATS}

\subsection{Caractérisation de l'échantillon étudié}

Tous les participants, à l'exception d'un, ont été capable de raconter l'histoire de la mise en place de leur portage de repas. Celui dont le récit n'était pas suffisamment précis a été exclu de l'analyse.

Le tableau 1 présente les caractéristiques des participants retenues pour l'analyse des données. Les participants inclus étaient âgés de 70 à 97 ans, avec une majorité de femmes et une large Tableau 1. Caractérisation de l'échantillon étudié.

\begin{tabular}{|c|c|c|c|c|}
\hline & $\begin{array}{c}\text { Total } \\
(n=30)\end{array}$ & $\begin{array}{c}\text { Clients } \\
\text { CASVP } \\
(n=15)\end{array}$ & $\begin{array}{l}\text { Clients } \\
\text { directs } \\
(n=15)\end{array}$ & p-value ${ }^{a}$ \\
\hline Genre, \% femmes & 21 & 11 & 10 & 1 \\
\hline Age moyen ${ }^{\mathrm{b}}$ & $85(8)$ & $83(8)$ & $87(8)$ & 0,24 \\
\hline \multicolumn{5}{|l|}{ Situation maritale } \\
\hline Célibataire / Séparé(e) & 15 & 9 & 6 & \multirow{3}{*}{0,27} \\
\hline Veuf / veuve & 14 & 5 & 9 & \\
\hline En couple & 1 & 1 & 0 & \\
\hline \multicolumn{5}{|l|}{ Niveau d'éducation } \\
\hline Aucun & 3 & 1 & 2 & \multirow{4}{*}{0,77} \\
\hline Primaire & 6 & 4 & 2 & \\
\hline Secondaire & 10 & 5 & 4 & \\
\hline Supérieur & 12 & 5 & 7 & \\
\hline \multicolumn{5}{|l|}{ Auto-perception du statut financier } \\
\hline Bas & 14 & 9 & 5 & \multirow{3}{*}{$0.06\left(^{*}\right)$} \\
\hline Moyen & 12 & 6 & 6 & \\
\hline Elevé & 4 & 0 & 4 & \\
\hline \multicolumn{5}{|l|}{ Historique du portage } \\
\hline Nombre de repas livrés par semaine ${ }^{\mathfrak{b}}$ & $8(4)$ & $10(4)$ & $6(2)$ & $0,01 * *$ \\
\hline $\begin{array}{l}\text { Durée depuis la première livraison (en } \\
\text { années) }\end{array}$ & $2,5(2)$ & $2(1)$ & $3(2)$ & 0,23 \\
\hline Prix & $9(4)$ & $6(3)$ & $11(4)$ & $4.10^{-4 * *}$ \\
\hline \multicolumn{5}{|l|}{ Statut cognitif } \\
\hline Score MMSE & $27(3)$ & $26(3)$ & $28(2)$ & 0,17 \\
\hline
\end{tabular}


${ }^{a} p$-value, test $t$ de Student (variables continues) ; $\left.{ }^{*}\right): p<0.1 ;{ }^{*}: p<0,05 ;{ }^{* *}: p<0,01$, test exact de Fisher (variables catégorielles) : comparaison des clients directs de la société de portage aux clients de la mairie de Paris. ${ }^{D}$ Moyenne (Déviation Standard)

majorité de personnes seules (célibataires, séparé.e.s ou veuf.e.s). De façon attendue, l'autoperception du statut financier des 'clients directs' de la société de portage est plus élevée que les 'clients CASVP'. Cependant le prix des repas étant plus élevé en clientèle directe qu'en clientèle CASVP (11€ en moyenne par repas pour les 'clients directs' contre $6 €$ en moyenne par repas pour les 'clients CASVP' ; $p<0.01$ ), les clients directs se faisaient en moyenne livrés moins de repas que les clients CASVP (6 repas en moyenne par semaine pour les 'clients directs' contre 10 repas en moyenne pour les 'clients CASVP' ; $p<0.01$ ). Aucune autre différence significative entre les deux groupes de clients n'a été retrouvée en ce qui concerne le genre, l'âge, la situation maritale, le niveau d'étude ou encore la durée depuis laquelle les sujets reçoivent le portage de repas (Tableau 1).

\subsection{Installation du service de livraison de repas}

La mise en place d'un service de livraison de repas s'est fait plus ou moins tôt dans les trajectoires de vie des participants. Ils étaient âgés de 65 et 94 ans lorsque leur livraison débutait. Le moment où le service de livraison est mis en place dépend de l'apparition des difficultés à réaliser les tâches en lien avec l'alimentation, ainsi que des ressources disponibles pour contourner ces difficultés, mais aussi de l'accès (ou non) à l'information concernant l'existence de service de livraison de repas à domicile.

\subsubsection{Les difficultés rencontrées par les personnes âgées}

Une large majorité des personnes interrogées a évoqué des difficultés associées à une diminution de leur capacité à faire les courses (troubles de l'équilibre, peur de tomber, difficulté à marcher) : "Je ne peux pas marcher toute seule: comment je fais les courses alors? » Madame T ; "J'ai eu du mal pour marcher, je suis tombée. Donc je ne sortais plus et j'ai pensé que ce serait beaucoup plus simple de me faire porter les repas. » Madame E. Certains participants ont également évoqué leur épuisement physique, leur difficulté à porter des sacs («De porter les trucs ça me fatigue!» Monsieur I), leur difficulté à pousser un chariot ("Je ne pouvais plus pousser le chariot. C'était fini, c’était pas possible. Le chariot, même s'il y avait une bouteille, je ne pouvais déjà plus. » Madame D). L'étude de Frongillo et al., (2010) traduit les mêmes difficultés à réaliser soi-même son approvisionnement chez les 
personnes recevant le portage de repas de la ville de New-York. Sur un échantillon de 1505 bénéficiaires interrogés, la moitié se faisait également aider pour les courses (23).

Quelques participants ont évoqué leur difficulté à cuisiner: "J'aime bien cuisiner, mais maintenant je peux plus parce que la plupart du temps je fais brûler les trucs [rires]. Ou je m'endors dans le fauteuil!» Madame B. Deux hommes ont évoqué ne pas avoir la compétence nécessaire en cuisine : «Les problèmes pour moi ont commencé à la mort de ma femme. [...] Je me débrouillais comme je pouvais, j'ai fait des expériences. [...] Puis je me suis rendu compte que je mangeais toujours la même chose. En plus, je ne suis pas du tout doué pour ça.» Monsieur F; "J'étais le meilleur ouvreur de boîtes de conserve du département! » Monsieur E.

\subsubsection{Le contexte d'installation du portage de repas}

Pour un tiers des participants, le service de livraison de repas a été mis en place de façon relativement soudaine, alors que ces personnes ne recevaient aucune aide auparavant. Dans ce contexte, la mise en place de ce service a été proposée au cours d'une hospitalisation, souvent en association avec d'autres aides. Autrement dit, la livraison de repas fait partie d'un arsenal d'aides mises en place pour prendre en charge une situation de dépendance sévère. Les participants dont la mise en place des aides a été soudaine étaient souvent parmi les moins entourés (sans enfant, famille peu proche...). Parfois c'est seulement après plusieurs hospitalisations que les aides ont été déployées et il est légitime de se demander si ces aides n'auraient pas pu être proposées lors de la première hospitalisation. Voici deux parcours qui illustrent une mise en place soudaine d'aides à domicile :

- Monsieur A : Cet homme de 81 ans vit seul et connait plusieurs soucis de santé depuis quelques années (infections urinaires, pulmonaires). En 2016, il perd connaissance dans la rue et est hospitalisé pour un pontage coronarien. C'est lors de cette hospitalisation que l'assistante sociale met en place une livraison de repas à domicile ainsi qu'un service d'aide-ménagère pour les courses et le ménage.

- Madame G : Cette femme de 79 ans souffre d'arthrose qui altère sa capacité à se déplacer depuis plusieurs années. Il y a trois ans, elle arrête de s'alimenter. Son fils, inquiet, la fait hospitaliser. Au cours de cette hospitalisation, une assistante sociale met en place un service de livraison de repas ainsi qu'une aide-ménagère pour les 
courses et le ménage, une aide-soignante pour la toilette et un service de téléassistance.

Pour la moitié de l'échantillon, la livraison de repas s'ajoutait ou remplaçait une partie des aides qui avaient été mises en place auparavant. Les personnes recevaient déjà de l'aide pour les courses et plus rarement pour la préparation des repas. Cette aide était apportée par un membre de leur famille, un voisin ou une aide-ménagère et était très souvent conservée après la mise en place du portage de repas comme complément d'approvisionnement. Les participants dont la mise en place des aides était progressive étaient bien entourés (bon relationnel avec leurs enfants, amis, voisins). Dans ce contexte, la mise en place d'un service de livraison s'inscrivait dans une aggravation progressive de la dépendance, comme l'illustrent ces deux exemples :

- Madame I : Cette femme de 72 ans souffre d'arthrose depuis la cinquantaine. Ces trois dernières années, elle a de plus en plus de mal à se déplacer. Elle commence par recevoir l'aide d'une femme de ménage, puis sa fille vient l'aider pour les courses. Il y a deux ans, un service d'aide-ménagère est mis en place pour les courses. Rapidement, sa fille décide de mettre également en place un service de livraison de repas.

- Madame J : Cette femme de 78 ans présente plusieurs pathologies: arthrose, diabète, dépression. On lui pose une première prothèse du genou à 58 ans, puis une seconde à 68 ans. Elle a toujours eu une femme de ménage, mais depuis quelques années, des amis l'aident pour les courses. En 2017, elle se rend à la mairie pour bénéficier de l'Allocation Personnalisée d'Autonomie (APA). Un service d'aideménagère est alors mis en place pour les courses et le ménage. Enfin, quand elle est hospitalisée à la suite d'un malaise il y a un an, une assistante sociale met en place un service de livraison de repas à domicile.

Il semble que cette installation progressive des aides soient plus courante pour les participantes de sexe féminin ainsi que pour les 'clients directs'.

La mise en place du portage des quelques participants restants ne s'inscrit pas dans les dynamiques décrites plus haut. C'est le cas de deux hommes Monsieur E et Monsieur D âgés respectivement de 70 et 81 ans pour qui cette livraison n'était pas indispensable, mais confortable. C'est la première aide qu'ils ont demandé et qui vient compenser un manque 
d'envie de faire : "Ça m'arrive d'acheter des choses [...] mais j'ai plus envie. » Monsieur $\mathrm{D}$; «C'est la fainéantise, la paresse et le confort quoi. » Monsieur E. C'est également le cas de Madame $\mathrm{S}$, pour qui la livraison de repas a été mise en place après la disparition de ses commerces de proximité. Cette femme de 77 ans, célibataire et sans enfant souffrait d'un handicap moteur et se faisait livrer ses courses par un magasin proche de chez elle. A la fermeture de ce magasin, elle a choisi de se faire livrer ses repas. C'est enfin le cas de Madame $\mathrm{O}$ : cette femme de 91 ans s'est toujours refusée à dépendre de ses enfants ou de la société : "Je voulais être en bonne santé physique pour ne pas être à charge de mes enfants. [...] Ne pas être à la charge des autres. » mais finit par se résoudre à installer une livraison de repas quand elle est devenue trop fatiguée pour faire ses courses.

\subsubsection{La source d'information}

L'accès à l'information de l'existence des services de livraison de repas est une condition clé pour mettre en place ce service. S'il apparaît que les services sociaux jouent un rôle important dans l'accès à cette information (notamment lors des hospitalisations), il existe d'autres sources d'information plus aléatoires ("bouche-à-oreille”, publicité).

Pour la moitié des participants, l'information concernant le service de portage de repas a été apportée par les services sociaux - les assistantes sociales de l'hôpital ou des mairies. C'est de fait la source d'information principale chez les participants bénéficiaires du service de portage de repas proposé par le Centre d'Action Sociale de la Ville de Paris ('clients CASVP'). Toutefois, certains 'clients directs' ont aussi reçu une information sur ce service par une assistante sociale, notamment au cours d'une hospitalisation.

- Madame C : Cette femme de 86 ans a subi plusieurs hospitalisations au cours des trois dernières années (pied cassé, escarre au talon, crise cardiaque). C'est au cours de la troisième hospitalisation qu'une assistante sociale lui propose de mettre en place un portage de repas à domicile.

- Monsieur H : Cet homme de 92 ans a différents problèmes de santé : insuffisance respiratoire, mobilité réduite. En 2016, il est hospitalisé pour une complication pulmonaire. Lors de ce séjour, une assistante sociale lui conseille de mettre en place la livraison de repas et lui fournit le contact de la société de portage. 
Lorsque l'aide aux courses est apportée par les enfants, c'était souvent ces derniers qui proposaient la mise en place d'un service de portage de repas à domicile à leurs parents.

- Madame M : A l'issue d'une première chute, cette femme de 84 ans se fait aider par sa fille pour faire les courses. En 2018, elle chute de nouveau et se fait hospitaliser. Lors de ce séjour à l'hôpital, sa fille découvre l'existence des services de livraison de repas à domicile, le propose à sa mère et fait les démarches pour le mettre en place.

Enfin, un tiers des participants, essentiellement les 'clients directs' ont découvert ce service grâce au « bouche à oreille » ou à la publicité.

- Madame L : Cette femme de 90 ans a découvert le service de livraison de repas tout à fait par hasard. Il y a 6 ans, alors qu'elle sortait encore, elle rencontre une dame sur un banc qui lui parle du portage de repas à domicile. Suite à cette rencontre, elle décide de mettre en place ce service en plus des aides qu'elle a déjà.

- Madame Q : Depuis une dizaine d'année, cette femme de 84 ans a des soucis pour se déplacer. Elle met en place les services d'une femme de ménage dont elle augmente progressivement le temps de travail. Après plusieurs chutes, elle ne peut plus sortir de chez elle. C'est à la télévision qu'elle entend parler de la société de portage de repas. Elle décide alors de s'inscrire pour de ne plus avoir à aller faire les courses.

\subsubsection{La capacité d'agir}

En ce qui concerne la capacité d'agir des participants, l'analyse des processus de mise en place d'un service de portage de repas ont permis d'identifier deux profils : un profil actif (17 participants) et un profil passif (13 participants).

Le profil passif qui inclut $3 / 4$ de 'clients CASVP' correspondait aux personnes qui ne se considéraient pas décisionnaires dans la mise en place du service de livraison de repas à domicile. Ces participants n'étaient à l'origine ni de la découverte de ce service, ni des démarches d'installation : "Mes enfants $m$ 'ont poussé à prendre les repas. C'est ma fille qui s'en est occupée. " Madame I. Pour un certain nombre de ces participants, le portage de repas leur était présenté comme incontournable : "C'était la seule, c'était la solution. Il existe pas 
non plus d'autres formules. » Monsieur B ; « [A l'hôpital], ils me laissaient entendre que ça n'irait pas en s'arrangeant. » Monsieur G.

Le profil actif qui inclut $3 / 4$ de 'clients directs' correspond aux personnes qui au contraire se sentaient décisionnaires dans la mise en place du service de livraison de repas. Elles ont été impliquées, au moins en partie, dans la prise de décision, la recherche d'information et la mise en place du service. Quand on demande à Madame A qui a pris la décision de mettre en place une livraison de repas à domicile, elle répond : "Ah c'est moi qui ai décidé ! ». Il est intéressant de noter que le niveau de dépendance des participants 'actifs' dans la mise en place du portage n'est pas forcément plus faible que les participants du profil passif.

- Madame K : Cette femme de 97 ans souffre d'arthrose depuis plus de 20 ans. Elle a également des problèmes de vue (DMLA) ainsi que des troubles de l'équilibre. Elle commence par se faire aider pour le repassage. Quand il devient difficile pour elle de sortir de chez elle, elle se fait aider par ses enfants pour les courses. Puis elle décide de faire une demande pour obtenir les aides sociales destinées aux personnes âgées dépendantes et s'adresse à la mairie. On lui propose alors de mettre en place un service de portage de repas et une aide-ménagère, qu'elle accepte. Récemment, à la suite d'une chute, elle souscrit à un service de téléassistance.

\subsection{Appropriation du service de livraison de repas}

En parallèle de l'histoire d'installation d'une livraison de repas à domicile, l'analyse des entretiens a permis de mettre en évidence trois stratégies principales mise en œuvre par les participants autant passifs que actifs pour garder une part de contrôle sur leur alimentation.

D'une part, la plupart des participants ont décidé et ajusté eux-mêmes le nombre et la nature des repas qu'ils souhaitent recevoir chaque semaine : "J'avais demandé des dîners au départ. Je suis passée aux déjeuners récemment, parce que j'ai vu que les menus étaient beaucoup mieux à midi que le soir. » Madame $\mathrm{H}$; «Enquêteur : En ce moment, vous avez six journées complètes [petit-déjeuner, déjeuner, dîner]. C'est ça? Enquêté : Oui. Avant je prenais que le repas du midi. Si ça va pas, et bien je prendrais un repas complet tous les deux jours. » Madame D ; "Au départ, j'ai pris toute la semaine, et puis maintenant je mange beaucoup moins. Alors j'ai supprimé deux jours. Et même encore là ... » Madame K. Certains revendiquent de ne se faire livrer que quelques repas par semaine pour conserver un espace de 
liberté sur les autres repas : "Cinq repas par semaine, c'est volontaire ! C'est tout simplement parce que ça me laisse une souplesse, une possibilité de faire ce que je veux. C'est-à-dire ou le restaurant, ou les surgelés [...] ou des copains. " Monsieur E; "De temps en temps, il vaut mieux se réserver un jour pour aller au restaurant, pour se payer un truc qu'ils peuvent pas nous donner. " Monsieur F. Afin de compléter la livraison de repas, certains participants font encore des courses dans des commerces proche de chez eux, mais la plupart se font aider. Les aliments achetés pour compléter la livraison de repas ne nécessitent généralement pas ou peu de préparation (œufs, jambon, salades, plats préparés...). Seuls quelques participants prenaient encore le temps de cuisiner : "Le soir, je me fais livrer des pommes de terre, des carottes, des courgettes et des tomates, je me fais une marmite de soupe, je les mets dans des récipients spéciaux et pendant 8 jours j'ai la soupe. » Monsieur C ; «[Je cuisine] moins qu'avant. Mais là par exemple je suis en train de faire de la confiture de groseille. » Monsieur I.

D'autre part, un tiers des participants ne consommaient pas le repas livré en une seule prise, mais répartissaient les aliments reçus sur plusieurs repas en les combinant avec des aliments achetés par ailleurs : "Généralement je mange pas tout à midi, alors je garde pour le soir mais je m'achète du taboulé, je m'achète des salades piémontaises ou des trucs comme ça. » Madame U; "Deux jours complets, ça fait quatre barquettes. Une barquette me fait deux repas, j'ai déjà huit repas! Il y a les entrées et les potages. Deux entrées, deux potages, ça fait encore deux repas. Un potage et le yaourt le soir ça suffit. » Madame E. Cependant la plupart des participants consommaient l'intégralité du repas livré en une seule prise : «[Le repas livré], je le prends que le midi hein. Ce sont des menus qui sont certainement étudiés pour une personne et je mange ce qu'on me donne. » Madame N.

Enfin, un tiers des participants a déclaré avoir réassaisonné les plats à son goût avec du sel, du poivre, mais aussi des condiments (moutarde, ketchup, huile d'olive, vinaigrette), des aromates (échalotes, ail, herbes...) : «J'ajoute des fois des condiments un peu, des choses, du sel ça arrive oui. Oui même du vinaigre, de la vinaigrette. » Monsieur F; "Je fais la grimace comme ça, je goûte un peu, oh c'est fade. Alors je mets du sel [...], je re-goûte... oh... je me dis drôlement bon! » Madame R. Un seul participant a déclaré recuisiner les plats livrés : « S'il y a du riz je fais recuire dans une poêle. C'est là que je mets de l'eau et un peu d'huile d'olive. " Madame B. 


\subsection{La satisfaction des participants vis-à-vis du portage}

Pour la plupart des participants, l'alimentation était une source de plaisir : "Je marche pas mais j'ai un bon appétit! » Madame N ; «Moi je mange pas par obligation, il faut manger par plaisir et il faut savourer ce qu'on mange. Vous voyez si c'est pour manger parce qu'il faut avaler, comme quand on était petit, non! Moi j'estime qu'à un certain âge, il faut qu'on se fasse plaisir et il faut savourer les choses de la vie. » Madame D ; «Moi comme on dit vulgairement, j'ai de la gueule. Donc j'aime bien manger, j'aime bien ce qui est bon.» Madame J.

Dans l'ensemble, les participants étaient plutôt satisfaits du service de livraison de repas (deux étaient très satisfaits ; 16 étaient satisfaits avec quelques critiques négatives). Seuls trois participants n'étaient pas du tout satisfaits du service de livraison. L'un de ces participants est un homme de 93 ans à qui la livraison de repas a été imposée. Il préférerait que ce soient ses enfants qui lui apportent ses repas (Monsieur C). Une autre participante très insatisfaite du service est une femme de 72 ans très sélective pour son alimentation et qui présente de nombreuses aversions. De ce fait, les repas qu'elle reçoit sont peu variés (Madame D). Enfin la troisième participante qui n'est pas du tout satisfaite de la livraison n'a plus beaucoup d'appétit et n'apprécie pas ce qui lui est livré (B10). De manière générale, la satisfaction des participants vis-à-vis du service de portage de repas était meilleure chez les 'clients directs' que chez les «clients CASVP ». De fait, les 'clients directs' bénéficient d'une offre plus large et plus personnalisée que celle proposée aux «clients CASVP». Les «clients directs» peuvent notamment choisir leurs menus et bénéficient d'un catalogue de recettes plus vaste que les «clients CASVP». Il est intéressant de noter que la satisfaction des participants du 'profil actif' n'est pas plus importante que celle des participants du «profil passif». Si certains «profils passifs » rejetaient ce service qui leur a été imposé (comme par exemple Monsieur C mentionné ci-dessus), d'autres s'en accommodaient. Ainsi, Madame H dont l'implication dans la mise en place du service de livraison de repas à domicile a été minime puisqu'elle a été faite par une assistante sociale, est très satisfaite de la prestation.

Les entretiens ont révélé plusieurs points de vigilance susceptibles d'influencer la satisfaction des bénéficiaires pour un service de livraison de repas. Un certain nombre de points concerne l'organisation du service : des horaires adaptés et respectés, des paniers repas complets et correspondant au menu annoncé et un interlocuteur clairement identifié au sein de la société : 
«Il faut qu'il y ait un intermédiaire qui peut écouter... Si c'est pour appeler un jour, avoir une personne et puis un autre jour en avoir une autre... Au final on n'est pas écouté ! " Monsieur B. Les autres points de vigilance concernent la qualité organoleptique des repas : le goût des plats, l'assaisonnement et la texture, notamment de la viande. En particulier, plusieurs participants ont évoqué une difficulté à mastiquer la viande à cause de troubles bucco-dentaires : "J'aurais aimé que la viande soit un peu plus tendre quelquefois. " Madame P : " J'ai un dentier en haut et en bas. J'ai que [les dents] de devant-là qui sont à moi et en haut j'en ai trois à moi. Alors on peut pas manger une viande qui n'est pas bien cuite... » Madame K.

Enfin, la grande majorité des participants ont décrit leur lien avec le livreur de façon très positive : «Il est adorable ce monsieur, il est gentil comme tout. » Madame $\mathrm{H}$; «Oh! Ils sont très, très gentils! » Madame A ; "Les livreurs sont très gentils, très polis, ah oui je n'ai rien à dire! » Madame N. Etant donné que toutes les personnes interrogées vivaient seules et sortaient dans l'ensemble peu de chez elles, leurs interactions avec leurs aidants - y compris le livreur de repas - jouent un rôle important dans leur vie sociale et le maintien d'un lien social.

\section{DISCUSSION}

\subsection{La mise en place d'une livraison de repas à domicile : un moment de rupture?}

La mise en place d'une aide à domicile, tel qu'un portage de repas, constitue une orientation particulière dans la trajectoire de vie des personnes âgées. Cet événement s'inscrit dans une dynamique de perte d'autonomie et vient modifier les rôles sociaux des participants. En effet, des personnes ayant toujours été responsables des activités alimentaires dans leur foyer se voient dépossédées des tâches qu'elles avaient l'habitude de réaliser. Cette situation renvoie à la notion de « déprise » décrite comme le réaménagement de l'existence avec l'avancée en âge. La personne qui devient dépendante acquiert un nouveau statut, celui d'aidé, et son identité peut en être ébranlée (24,25). De fait, c'est dans les actions et l'organisation du quotidien que l'identité d'une personne s'établit, qu'elle «se raconte » (25). En conséquence, la mise en place d'un portage de repas peut constituer un élément de rupture dans la trajectoire de vie des personnes âgées. Cependant, dans notre enquête, les participants ne l'ont pas décrit comme un événement marquant ayant bouleversé leur vie. Ce sont davantage les événements de santé, souvent à l'origine de la mise en place d'un portage de repas, qui sont 
vécus comme des moments de "rupture". Pour de nombreuses personnes interrogées, les aides à domicile se sont mises en place progressivement et le portage de repas intervient après plusieurs années où ils se font déjà aider. Cet événement n'est alors pas vécu comme une rupture dans leur trajectoire de vie, mais plutôt comme une étape parmi d'autres dans leur transition vers la dépendance.

\subsection{De l'autonomie vers la dépendance}

A la lumière de nos résultats, nous pouvons distinguer trois étapes lors de la mise en place d'un service de portage de repas à domicile : la prise de conscience des difficultés à réaliser certaines tâches du quotidien en relation avec l'acte alimentaire, la mise en place effective d'une aide pour pallier ces difficultés et l'appropriation de cette aide pour l'incorporer dans son "nouveau" quotidien.

Il n'est pas toujours aisé pour une personne âgée de prendre conscience de ses difficultés “à faire”. En effet, si les difficultés apparaissent progressivement, la personne va mettre en place des stratégies de compensation - va adapter ses habitudes de vie - pour s'en accommoder. Il peut alors être difficile pour elle de formuler clairement ses besoins et de mettre en place les aides appropriées. Par ailleurs, Cardon \& Gojard, (2009) ont souligné la difficulté à accepter de se faire aider pour des tâches dont on a eu la responsabilité tout au long de sa vie (13). Pour certaines personnes, le renoncement à ces tâches est si difficile qu'il peut conduire à des situations de «non-recours ». Ce non-recours est souvent accompagné de représentations négatives des services d'aide et de la situation d'aidé (5). C'est presque un acte de résistance vis-à-vis de la dépendance et cela peut expliquer pourquoi dans un certain nombre de cas la mise en place d'aides se fait tardivement au regard des difficultés rencontrées par la personne. C'est alors souvent une panoplie d'aides qui est attribuée à la personne, par exemple par une assistante sociale à la sortie d'une hospitalisation. Nous avons par ailleurs observé que pour les participants dont l'entourage familial et/ou amical était présent, la mise en place des aides s'est faite de façon progressive. De fait, Gagnon, (2018) souligne que plus le réseau d'une personne est étendu, plus elle y trouve les ressources nécessaires pour accepter de renoncer à certaines activités (25).

En ce qui concerne la mise en place du service de livraison de repas à domicile, notre enquête a révélé deux profils : un profil passif avec des personnes ne s'impliquant pas du tout dans la 
mise en place effective du service (prise d'information, démarches...) et un profil actif avec des personnes s'impliquant au moins en partie dans cette mise en place. Il ne semble pas que ce soit le degré d'investissement de la personne dans les activités alimentaires qui conditionne son profil : certaines personnes, très investies dans les courses ou la cuisine au cours de leur vie, ne se sont pas impliquées dans la mise en place du service de portage. A l'inverse, certaines personnes peu investies dans les activités alimentaires ont été parties prenantes dans la mise en place de ce service. Nous pouvons aussi faire l'hypothèse que le degré d'implication d'une personne dans la mise en place d'une aide dépend de la "capacité à agir" ("human agency") de cette personne (20). Cette "capacité à agir" qui renvoie à la capacité d'une personne d'influer sur sa trajectoire de vie s'acquiert au cours de la vie, en fonction des opportunités, des contraintes, des ressources et des expériences vécues (5). Bien que certaines personnes devenaient très dépendantes pour les tâches de la vie quotidienne, elles conservaient une “capacité à agir" en étant moteur du processus d'installation des aides.

Cette volonté de rester acteur de son alimentation transparaît également dans les stratégies d'appropriation du service de livraison mises en œuvre par les personnes interrogées. Lorsqu'une personne âgée délègue les courses et la préparation de ses repas à un tiers (en l'occurrence, un service de livraison de repas), elle passe du statut de "faiseur" au statut "d'aidé". Cette situation de déprise, « évoque un renoncement, une perte de capacité, une réduction des possibles, en même temps qu'elle suggère une reprise en main, une capacité d'adaptation à sa nouvelle situation ; elle renvoie à une certaine maitrise par la personne sur son existence, alors même que ses forces déclinent »(25). La personne doit trouver un équilibre entre délégation et contrôle (15). De fait, les résultats de notre enquête ont montré que certains participants choisissent volontairement de ne prendre qu'un repas livré par jour afin de pouvoir choisir les aliments consommés aux autres repas. Un résultat similaire avait été observé par Frongillo et al., (2010) : ces auteurs avaient observé que les 2/3 des bénéficiaires du service de livraison de repas fourni par de la ville de New-York ne consommaient pas exclusivement des repas livrés mais continuaient de préparer eux-mêmes certains de leurs repas (23).

En conclusion de ces paragraphes, on peut se demander si le fait qu'une personne s'implique dans la mise en place du service et garde un espace de contrôle sur son repas (réassaisonnement, choix des menus, courses complémentaires) influence l'acceptation de cette 
personne pour ce service. Deux exemples peuvent illustrer cette interrogation. Monsieur C, 93 ans, est tout à fait conscient de ne plus avoir les mêmes capacités physiques qu'autrefois. Il explique qu'il ne parvient plus à réaliser certaines tâches du quotidien. Cependant il a beaucoup de mal à accepter d'être aidé. Il accepte mal la livraison de repas à domicile et aurait préféré continuer à être aidé par ses enfants. Cette difficulté à accepter la dépendance et la mise en place d'aides se cristallisent dans une insatisfaction vis-à-vis des repas livrés. En ce qui concerne Madame S, 77 ans, elle est née avec un handicap physique. Elle a toujours fait appel à des aides extérieures pour réaliser certaines tâches de la vie quotidienne. Son parcours est singulier car elle n'a pas véritablement vécu une entrée dans la dépendance. Tout au long de sa vie, elle a eu à définir un espace de contrôle pour trouver sa place en tant que personne aidée. C'est elle qui a pris la décision de mettre en place un service de livraison et qui a fait les démarches. Elle se déclare très satisfaite de cette solution, en acceptant que tout ne peut pas être toujours. De façon intéressante, Frongillo et al., (2010) ont montré que les personnes les plus satisfaites de leur portage de repas étaient aussi celles qui avaient une meilleure santé mentale et émotionnelle et étaient bien entourées (23).

\subsection{La place des services de livraison de repas dans les dispositifs d'accompagnement de la personne âgée à domicile}

Plusieurs études ont montré que la mise en place d'un service de portage de repas à domicile permettait d'améliorer le statut nutritionnel des personnes âgées (26-29). Ainsi, la livraison de repas 4 à 7 jours par semaine pendant trois mois a entraîné une augmentation du poids significative chez les personnes recevant ces repas par rapport à un groupe contrôle ne bénéficiant pas de portage de repas à domicile (28). Dans cette étude, les repas livrés étaient préparés de façon à respecter les recommandations nutritionnelles en optimisant la qualité sensorielle des plats (produits frais). R. An, (2015) a montré que, les personnes qui bénéficiaient d'une livraison de repas à domicile, consommaient davantage de protéines les jours où elles recevaient un repas que les jours où elles préparaient leurs repas elles-mêmes (26). Enfin, Arjuna et al., (2018) ont montré que la livraison d'au moins trois déjeuners enrichis en protéines et en énergie par semaine entraînait une diminution du risque de dénutrition. La dénutrition correspond à un déséquilibre des apports nutritionnels par rapport aux besoins de l'organisme (27). Ce déséquilibre conduit à un amaigrissement, à une diminution des réserves musculaires et à une altération des défenses de l'organisme. Sans 
prise en charge, la dénutrition induit ou aggrave un état de fragilité et de dépendance, et affecte la qualité et l'espérance de vie des personnes (30,31). En conséquence, le portage de repas pourrait être un outil intéressant pour prévenir la dénutrition au sein de la population âgée et ainsi limiter la perte d'autonomie dans le temps. En parallèle, le portage de repas permet également d'entretenir un certain lien social, à travers celui qui se crée entre le bénéficiaire et le livreur. Presque tous les participants de notre étude ont décrit avoir de bons rapports avec leur livreur. De même, Frongillo et al., (2010) ont observé que 50\% des bénéficiaires d'un service de livraison de repas à New-York rapportaient parler régulièrement avec leur livreur (23).

Pour un tiers des participants, l'hospitalisation a été un élément clef dans la mise en place d'un portage de repas. Pour certains participants, cette mise en place semblait relativement tardive, notamment lorsqu'elle survenait après plusieurs hospitalisations. De fait, la mise en place d'un portage de repas se heurte à deux freins. D'une part, le "non-recours", évoqué plus haut, qui peut occasionner une mise en place tardive de ce service. D'autre part, la "nonconnaissance" de l'existence de ce type de service (5). En effet, notre enquête a révélé le caractère parfois aléatoire de la façon dont les participants avaient eu connaissance de ce service (bouche-à-oreille, publicité). Ceci pose la question des personnes qui pourraient bénéficier de ce service mais qui n'y ont pas recours faute d'avoir accès à l'information. Il serait sans doute nécessaire d'améliorer la visibilité de ce type de service afin que les personnes qui en ont besoin puissent avoir l'opportunité de le mettre en place de manière préventive plutôt qu'en situation d'urgence. En particulier, on peut questionner le rôle des professionnels de santé (médecins, pharmaciens) : dans notre enquête, ils ont parfois été cités comme soutien à la décision de mettre en place un portage de repas, mais jamais comme prescripteurs.

Enfin, une meilleure acceptation de ce service - et des services d'aide en général, passe sans doute par une modification du regard de la société sur les personnes âgées : passer d'une image négative voire dégradante de ces aides renvoyant à un corps qui vieillit et des capacités qui diminuent, à une image plus positive renvoyant plutôt au maintien d'une bonne qualité de vie et à un certain confort. 


\section{CONCLUSION}

Il existe différents processus de mise en place d'une livraison de repas dans la trajectoire de vie d'une personne âgée. Ils s'inscrivent majoritairement dans une situation de perte d'autonomie dans les activités alimentaires, notamment quand l'approvisionnement devient compliqué à réaliser. Le contexte de vie de la personne âgée ainsi que son entourage influencent fortement la dynamique de mise en place de ce service à domicile, qui sera ainsi plus ou moins progressive. Le portage de repas à domicile est souvent associé à une image négative : celle du “je ne peux plus faire”. Il mériterait d'être mieux valorisé comme une aide "qui me facilite la vie" et "qui me permet de rester chez moi”. La revalorisation du portage de repas à domicile passe aussi probablement par l'introduction d'espaces de liberté dans ce service (personnalisation des prestations, introduction et renforcement des espaces de choix choix du menu, de l'heure de livraison), au regard des trajectoires et des habitudes de vie très variées des bénéficiaires.

\section{Protection des droits des sujets humains et animaux}

Les auteurs déclarent que les travaux décrits n’ont pas impliqué d'expérimentations sur les patients, sujets ou animaux.

\section{Consentement éclairé et confidentialité des données}

Les auteurs déclarent avoir obtenu le consentement éclairé écrit des patients et/ou des sujets auxquels ils font référence dans cet article et déclarent également que l'article ne contient aucune donnée personnelle pouvant identifier le patient ou le sujet.

\section{Déclaration de liens d'intérêts}

Les auteurs ont déclaré le(s) lien(s) d'intérêt suivant(s) : ce travail a été réalisé dans le cadre de la convention CIFRE n 1576 , le contrat doctoral de Ségolène Fleury a ainsi été financé par la société Saveurs et Vie, dont Paul Tronchon et Stéphanie Roux sont membres. L'encadrement académique de cette thèse est réalisé par Claire Sulmont-Rossé et Virginie Van Wymelbeke.

\section{Financement}


Cette étude a été financée par : cette étude fait partie du projet RENESSENS, financé par l'Agence régionale de la recherche (ANR-13-ALID-0006-02).

\section{Contribution et responsabilité des auteurs}

L'ensemble des auteurs attestent du respect des critères de l'International Committee of Medical Journal Editors (ICMJE) en ce qui concerne leur contribution à l'article.

L'ensemble des auteurs attestent du respect des critères de l'International Committee of Medical Journal Editors (ICMJE) en ce qui concerne leur contribution à l'article. Les contributions des auteurs se sont réparties comme suit : Ségolène Fleury : conceptualisation, recherche, fourniture de ressources, conservation des données, rédaction - version originelle, visualisation, supervision Claire Sulmont-Rossé-Validation, révision et correction, visualisation, obtention du financement Paul Tronchon - Révision et correction, obtention du financement Stéphanie Roux - Révision et correction Virginie Van Wymelbeke - Validation, révision et correction, obtention du financement.

\section{Remerciement}

Cette étude fait partie du projet RENESSENS, financé par l'Agence nationale de la recherche (ANR-13-ALID-0006-02). Ce travail été réalisé avec l'aide de Claire Manière, Marion Perruchaud, Charlotte Meunier, Juliane Rota et Anaïs Roger respectivement en appuis pour le recrutement, les entretiens et l'analyse de la satisfaction des participants. Enfin, les éléments de réflexion apportés par Didier Vinot, Fabien Canolle, Pierrine Didier, Isabelle Maître et Agathe Raynaud-Simon ont été précieux pour mener à bien cette étude.

\section{Références}

(1) N. Blanpain and O. Chardon, Projections de population à l'horizon 2060. Un tiers de la population âgé de plus de 60 ans, 2010, 5.

(2) A. Lecroart, O. Froment, C. Marbot and D. Roy, Projection des populations âgées dépendantes, Solidarité \& Santé 43, 2013, 28. 
(3) F. Martial, I. Naudy-Fesquet, P. Roosz and J. Tronyo, Tableaux de l'économie française. Institut national de la statistique et des études économiques, 2015, CERTU; Paris, France, [Collection INSEE Références].

(4) O. Messiaen, $8^{\circ}$ débat OCIRP autonomie. Perte d'autonomie : quelles politiques, quels financnements ?, 2016, Maison de la radio; Paris.

(5) B. Masotti, Demander (ou pas) l'aide à domicile au grand âge. L'agency des personnes âgées, Gerontol Soc 40 (3), 2018, 79.

(6) A. Warde and K. Hetherington, English households and routine food practices: a research note, Sociol Rev 42 (4), 1994, 758-778.

(7) A. Warde, S.-L. Cheng, W. Olsen and D. Southerton, Changes in the practice of eating: a comparative analysis of time-use, Acta Sociologica 50 (4), 2007, 363-385.

(8) C. Pettinger, M. Holdsworth and M. Gerber, Meal patterns and cooking practices in Southern France and Central England, Public Health Nutr 9 (8), 2006, 1020-1026.

(9) B. Hale, P. Barrett and R. Gauld, The age of supported independence, 2010, Springer Netherlands; Dordrecht, [cited 2020 Jun $11 . \quad$ Disponible sur : http://link.springer.com/10.1007/978-90-481-8814-7].

(10) B. Masotti, Faire l'épreuve de l'aide à domicile au grand âge [Sciences Sociales], 2016, Univesité de Genève.

(11) J.-M. Vienne, Qu'est-ce que l'intimité ? de l'ouvrage L'intimité menacée ?, Enjeux éthiques dans la pratique du soin et de l'accompagnement, 2019, ERES 2019.

(12) M. Winance, Dépendance versus autonomie. De la signification et de l'imprégnation de ces notions dans les pratiques médicosociales, Sci Soc Sante 25 (4), 2007, 83-91.

(13) P. Cardon and S. Gojard, Les personnes âgées face à la dependance culinaire : entre delegation et remplacement, 2009, 26.

(14) P. Cardon, Vieillissement et délégation alimentaire aux aides à domicile : entre subordination, complémentarité et substitution, 2007, 28. 
(15) P. Cardon, Regard sociologique sur les pratiques alimentaires des personnes âgées vivant à domicile, Gerontol Soc 33 (134(3)), 2010, 31.

(16) S. Gojard and A. Lhuissier, Monotonie ou diversité de l'alimentation : les effets du vieillissement. Monotonie ou diversité de l'alimentation : les effets du vieillissement, 2003.

(17) I. Maitre, V. Van Wymelbeke, M. Amand, E. Vigneau, S. Issanchou and C. SulmontRossé, Food pickiness in the elderly: relationship with dependency and malnutrition, Food Quality and Preference 32, 2014, 145-151.

(18) E. Maurel, A. Boulegue and L. Faibis, Le marché du portage de repas à domicile à l'horizon 2020 : pressions sur les prix, nouveaux entrants, montée en gamme de l'offre : quels leviers et perspectives de croissance ?, 2018, Xerfi; Paris.

(19) C. Bardez, M. Cressens, A. Hilaire, S. Lyannaz, J. Montignies, B. Costa, et al., Personnes âgées vivant à domicile, le portage de repas : élément essentiel de lutte contre la dénutrition? Module interprofessionnel de santé publique, École des Hautes Études en Santé Publique (EHESP), 2008, EHESP (École des Hautes Études en Santé Publique).

(20) S. Hitlin and G.H. Elder, Time, self, and the curiously abstract concept of agency, Sociol Theory 25 (2), 2007, 170-191.

(21) M.F. Folstein, S.E. Folstein and P.R. McHugh, Mini-mental state, J Psychiatr Res 12 (3), 1975, 189-198.

(22) P. Antoine, X. Bry and P.D. Diouf, La fiche AGEVEN, un outil pour la collecte des données rétrospectives, 1987.

(23) E.A. Frongillo, T.D. Isaacman, C.M. Horan, E. Wethington and K. Pillemer, Adequacy of and satisfaction with delivery and use of home-delivered meals, J Nutr Elder 29 (2), 2010, 211-226.

(24) P. Ancet, Identité narrative, déprise et vécu du vieillissement, Gerontol Soc 40 (1551), $2018,45$.

(25) É. Gagnon, La déprise comme interrogations : autonomie, identité, humanité, Gerontol Soc 40 (1551), 2018, 33. 
(26) R. An, Association of home-delivered meals on daily energy and nutrient intakes: findings from the national health and nutrition examination surveys, J Nutr Gerontol Geriatr 34 (2), 2015, 263-272.

(27) T. Arjuna, M. Miller, S. Soenen, I. Chapman, R. Visvanathan and N.D. LuscombeMarsh, Serve size and estimated energy and protein contents of meals prepared by meals on wheels' South Australia Inc.: findings from a meal audit study, Foods 7 (2), 2018, 26.

(28) K.F.M. Denissen, L.M.J. Janssen, S.J.P.M. Eussen, M.C.J.M. van Dongen, N.E.G. Wijckmans, N.D.M. van Deurse, et al., Delivery of nutritious meals to elderly receiving home care: feasibility and effectiveness, J Nutr Health Aging 21 (4), 2017, 370-380.

(29) M.F. O'Leary, M. Barreto and J.L. Bowtell, Evaluating the effect of a home-delivered meals service on the physical and psychological wellbeing of a UK population of older adults - A pilot and feasibility study, J Nutr Geront Geriatr 39 (1), 2020, 1-15.

(30) M. Ferry, E. Alix, P. Brocket, T. Constans, B. Lesourd, P. Pfitzenmeyer, et al., Elsevier for mortality in hospitalised patients: the NutritionDay survey - Conséquences globales de la dénutrition, Clin Nutr 4 (28), 2012, 484-491.

(31) M. Hiesmayr, K. Schindler, E. Pernicka, C. Schuh, A. Schoeniger-Hekele, P. Bauer, et al., Decreased food intake is a risk factor for mortality in hospitalised patients: the NutritionDay survey 2006, Clin Nutr 28 (5), 2009, 484-491 